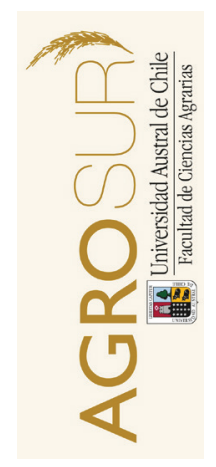

\title{
Escarabajos estercoleros nativos en Chile. Una revisión con énfasis en su ecología
}

\author{
Native dung beetles in Chile. A review with emphasis in their ecology \\ González-Chang, M. ${ }^{a, b, c *}$, Carrillo, R. ${ }^{d}$, Pinochet, D. ${ }^{b, e}$ \\ ${ }^{a}$ Bio-Protection Research Centre, Lincoln University, Ellesmere Junction/Springs Road, Lincoln, Nueva Zelanda. \\ ${ }^{b}$ Centro de Investigación en Suelos Volcánicos, Universidad Austral de Chile, Independencia 641, Valdivia, Chile. \\ ${ }^{c}$ Instituto de Ecología y Biodiversidad, Las Palmeras 3425, Santiago, Chile. \\ ${ }^{d}$ Instituto de Producción y Sanidad Vegetal, Universidad Austral de Chile, Independencia 641, Valdivia, Chile. \\ ${ }^{e}$ Instituto de Ingeniería Agraria y Suelos, Universidad Austral de Chile, Independencia 641, Valdivia, Chile.
}

\begin{tabular}{l} 
A R T I C L E I N F O \\
\hline Article history: \\
Received 29.04.15 \\
Accepted 22.11.15 \\
\hline Keywords: \\
Livestock production \\
Insect conservation \\
Ecosystem services \\
Coprophagous beetles \\
\hline Review Article, \\
Soil Science \\
\hline *Corresponding author: \\
Mauricio González-Chang \\
E-mail address: \\
mauriciogonzalezchang@gmail.com
\end{tabular}

\section{A B S T R A C T}

Dung beetles are crucial on the performance of different ecosystems, promoting several ecosystem services. In livestock production, these services are mainly related to the enhancement of soil fertility and the indirect biological control of parasites, through dung burial. Despite their known importance worldwide, in Chile the available scientific studies related to their ecology are scarce, despite the diversity of species found along the country. This work is an historical review of the available studies in Chile related to dung beetles, with emphasis in the advances on their ecology. Based on current knowledge, the potential of these insects for the improvement of the sustainability of livestock production in southern Chile is suggested.

\section{RESUMEN}

Los escarabajos estercoleros son determinantes en el funcionamiento de distintos ecosistemas, promoviendo diversos servicios ecosistémicos. En los sistemas ganaderos, estos servicios están relacionados principalmente en el aumento de la fertilidad del suelo y el control biológico indirecto de parásitos, a través del entierro del estiércol. A pesar de su reconocida importancia a nivel mundial, en Chile existen escasos estudios sobre su ecología, no obstante que la diversidad de especies se distribuye a lo largo de todo el país. Este trabajo es una revisión histórica de los estudios existentes en Chile en relación a estos escarabajos estercoleros, dando énfasis a los avances en su ecología. Basado en el conocimiento actual de este gremio, se sugiere el potencial de estos insectos para mejorar la sustentabilidad del manejo ganadero en el sur de Chile.

Palabras clave: Producción ganadera, conservación de insectos, servicios ecosistémicos, escarabajos coprófagos.

\section{INTRODUCCIÓN}

La ganadería en Chile presenta una variada distribución, con diferentes rubros productivos a lo largo del país. En la zona sur, se concentra la producción bovina para carne y leche, mientras que en la zona austral, predomina la producción ovina para carne y lana. Debido a la importancia, tanto cultural como económica, que estas actividades productivas tienen en el país, es necesario poder estudiar formas de ganadería que sean ecológicamente viables. Esto podría contribuir a reducir la cantidad de insumos utilizados en la producción animal, lo que se traduce en beneficios económicos hacia el agricultor, y al mismo tiempo, promueve diversos servicios ecosistémicos sobre las comunidades aledañas. En este sentido, la comunidad de escarabajos estercoleros presente en estos agroecosistemas juega un papel importantísimo sobre la producción y regulación de éstos (Nichols et al., 2008). Por esta razón, el presente trabajo tiene como objetivo ser una revisión histórica no-sistemática de las publicaciones realizadas en Chile en relación a comportamiento, biología y ecología de escarabajos estercoleros nativos, sin pretender ser una revisión sistemática del estado del arte en eco- 
logía de escarabajos estercoleros a nivel internacional. No obstante lo anterior, se presentan generalidades en relación al comportamiento y a las funciones ecológicas que proveen estos insectos, para luego presentar los estudios realizados en Chile en relación a especies nativas. Finalmente, y basado en el conocimiento actual de este grupo de insectos, se sugiere el potencial de éstos en la sustentabilidad de la producción ganadera en el sur de Chile, proponiendo desafíos futuros para promover el precario desarrollo en esta área de la entomología aplicada y la ganadería sustentable en Chile.

\section{ESCARABAJOS ESTERCOLEROS. GENERALIDADES}

Los escarabajos estercoleros (Coleoptera: Scarabaeoidea) derivan su nombre de la relación que mantienen con las fecas de los animales; las cuales utilizan para su alimentación y para proveer alimento a su descendencia. Existen tres diferentes comportamientos que presentan estos escarabajos en relación a la utilización y reubicación de la feca animal (Halffter y Edmonds, 1982; Nichols et al., 2008); 1) paracópridos ("tunnelers"), los cuales construyen túneles de nidificación, de diversa complejidad arquitectónica, inmediatamente debajo o al lado de su fuente de alimento (Figura 1a); 2) telecópridos ("rollers"), quienes transportan horizontalmente una esfera de estiércol desde su fuente de alimento, construyendo su nido a cierta distancia del origen de ésta (Figura 1b); y 3) endocópridos ("dwellers"), los cuales construyen su nido al interior de su fuente de alimento, no existiendo reubicación del estiércol luego de ser colonizado, como en los casos anteriores (Figura 1c) (Halffter y Edmonds, 1982; Nichols et al., 2008).

El comportamiento que presentan estos insectos genera una serie de beneficios ecológicos, entre los que se mencionan el reciclaje de nutrientes, la bioturbación, la estimulación del crecimiento vegetal, la dispersión secundaria de semillas, la supresión de parásitos (tanto internos como externos), la regulación trófica e incluso la polinización (Nichols et al., 2008). Debido a su importancia, es que durante los últimos 30 años se han realizado importantes avances científicos concernientes a su ecología y evolución, recopilados en Halffter y Edmonds (1982), Hanski y Cambefort (1991), Scholtz et al. (2009), Simmons y Ridsdill-Smith (2011), permitiendo utilizarlos como modelos biológicos en distintos experimentos alrededor del mundo. Desde un punto de vista agroecológico, estos insectos contribuyen, entre otros, con el aumento en la fertilidad del suelo (v.g., Lastro, 2006) y la reducción de parásitos que afectan al ganado bovino (v.g., Edwards y Aschenborn, 1987). En Estados Unidos, se ha estimado su contribución a los sistemas ganaderos en 380 millones de dólares al año (Losey y Vaughan, 2006). Sin embargo, esta contribución depende de su adaptación al estiércol de vacuno, ya que algunas especies son atraídas por determinados

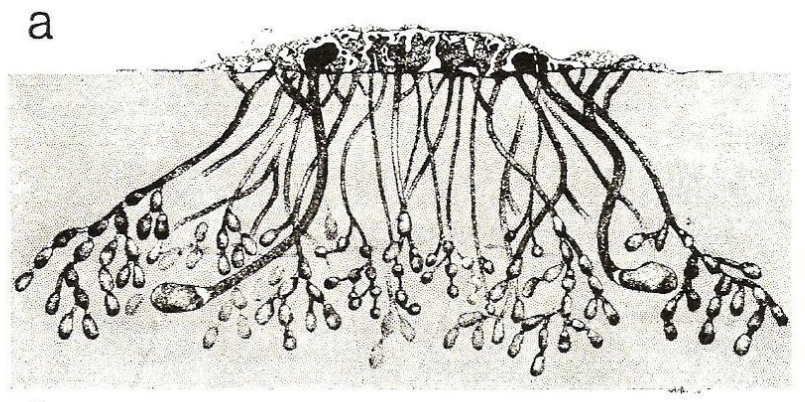

b
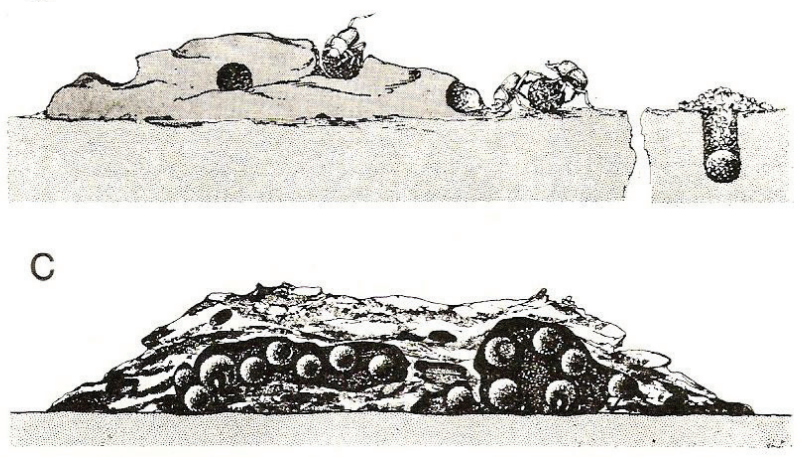

Figura 1. Diferentes comportamientos presentes en escarabajos estercoleros en relación a la utilización de su fuente de alimento. [a] Paracópridos, [b] Telecópridos y [c] Endocópridos. Adicionalmente, se muestra una representación general de la forma y ubicación de sus bolas de crianza o crotovinas para cada caso. Fuente: (Halffter \& Edmonds, 1982, adaptado de Bornemissza, 1976).

Figure 1. Different behaviors of dung beetles related to their food source utilization. [a] Paracoprids, [b] Telecoprids and [c] Endocoprids. In addition, the general shape and position of their brood balls or krotovinas are shown for each case. Source: (Halffter \& Edmonds, 1982, adapted from Bornemissza, 1976).

tipos de estiércol (especialistas), mientras que otras son atraídas por distintos tipos de estiércol (generalistas) (Philips, 2011). Por esta razón, es que ciertas especies nativas de escarabajos no son capaces de procesar estiércol proveniente de bovinos (Bos taurus (Linnaeus, 1758)), debido a la nula evolución entre ambos organismos. Bajo este concepto, parece factible la introducción de especies estercoleras exóticas que sean capaces de utilizar el estiércol bovino como sustrato, en zonas del planeta donde se requiera promover los servicios ecosistémicos producidos por la ganadería. Ejemplo de lo anterior ha sucedido en Australia desde la década de 1970, con notable aumento en la degradación del estiércol bovino tras la introducción y establecimiento de Digitonthophagus gazella (Fabricius, 1787) (Bornemissza, 1976). No obstante, existe evidencia que muestra la adaptación de escarabajos nativos a estiércol de bovino, aun en ausencia de este grupo de especies en términos evolutivos (v.g., Jones et al., 2012; González, 2013). Por esta razón, el estudio de la ecología y la diversidad de 
la comunidad estercolera nativa presente en distintos agroecosistemas es crucial al momento de implementar un programa que busque la sustentabilidad de la producción ganadera. Esto es de especial relevancia en los sistemas ganaderos del sur de Chile, considerando las especies allí presentes; especialmente si se piensa en la introducción de especies estercoleras exóticas, ya que hay evidencia de la competencia inter e intra específica que existe en estos insectos (Giller y Doube, 1989), lo cual podría afectar las comunidades estercoleras locales, como se ha visto en especies mesoamericanas (Horgan y Fuentes, 2005). En Chile, se ha realizado la introducción de dos especies estercoleras exóticas a Isla de Pascua durante 1988: Onitis vanderkelleni (Lansberge, 1886) y D. gazella, quienes disminuyeron la cantidad de moscas que afectaban tanto a humanos como a ganado bovino a fines de los años 80 (Ripa et al., 1995). Hasta la fecha, no existen estudios que muestren como esta introducción afectó a la especie Aphodius lividus (Olivier, 1789) (= Aphodius pseudolividus (Balthasar, 1941)), presente en Isla de Pascua en la década de 1970 (Campos y Peña, 1973). Sin embargo, existe evidencia reciente que sugiere una disminución de las comunidades estercoleras nativas en Colombia debido a la presencia del escarabajo exótico D. gazella (Noriega et al., 2011). Cabe destacar que el origen de D. gazella en América, se produjo tras su liberación en Texas, Estados Unidos en 1972 (Fincher et al., 1983). 40 años después, esta especie se encuentra ampliamente distribuida en todo el continente, registrándose en América del Sur en Brasil, Colombia, Paraguay, Bolivia, Argentina, Venezuela, Uruguay y Perú (Noriega et al., 2010), lo que muestra la facilidad que tiene este insecto para dispersarse y establecerse, afectando eventualmente a las comunidades estercoleras locales. Por esta razón, el propósito de este trabajo es recopilar la información actual relacionada con la ecología de escarabajos estercoleros nativos en Chile, con el objetivo de poder identificar futuros desafíos en relación a la conservación de estos insectos, con el fin de promover diversos servicios ecosistémicos en sistemas ganaderos del sur de Chile.

\section{ESPECIES NATIVAS EN CHILE}

En Chile, las especies estercoleras nativas se distribuyen desde Arica a Punta Arenas, presentando variadas formas, colores y comportamientos. De ellas, 4 especies son paracópridas: Frickius costulatus (Germain, 1897), Frickius variolosus (Germain, 1897), Homocopris torulosus (Eschscholtz, 1822) y Homocopris punctatissimus (Curtis, 1844); 5 especies telecopridas: Megathopa villosa (Eschscholtz, 1822), Scybalophagus rugosus (Blanchard, 1843), Tesserodoniella elguetai (Vaz de Mello y Halffter, 2006), Tesserodoniella meridionalis (Vaz de Mello y Halffter, 2006) y Taurocerastes patagonicus (Philippi, 1866). Los escarabajos con comportamiento endocóprido no han sido estudiados en Chile, en relación a su biología y ecología, presentándose solo registros anecdóticos. Se reporta a Podotenus fulviventris (Fairmaire y Germain, 1860) presente comúnmente en estiércol de vacuno y humano (Smith y Skelley, 2007), a A. pseudolividus en estiércol de caballo (Campos y Peña, 1973) y a Aphodius granarius (Linnaeus, 1767), en estiércol de vacuno, éste último presentando una distribución cosmopolita, no siendo nativo de Chile. Posiblemente, Ataenius chilensis (Solier, 1851), posea comportamiento endocóprido, basado en la cercanía taxonómica que posee en relación a otras especies encontradas en estiércol animal como Ataenius gracilis (Melsheimer, 1846) y Ataenius platensis (Blanchard, 1847) (Smith y Skelley, 2007). No obstante lo anterior, no existe hasta la fecha información sobre la biología y/o ecología en la mayoría de estas especies, por lo que para considerarlas especies endocópridas es necesaria más investigación. Adicionalmente, se desconoce absolutamente el ciclo de vida de especies endémicas como Amerisaprus valdivia (Stebnicka y Skelly, 2004), Acanthaphodius bruchi (Schmidt, 1909), Leiopsammodius indefensus (Schmidt, 1909), Orodaliscoides rugosiceps (Harold, 1859), Oxyataenius morosus (Harold, 1869), Odontopsammodius cruentus (Harold, 1868) y Symphodon anomalus (Harold, 1874). Algunas de estas especies son consideradas detritívoras, lo que sugiere que su mecanismo de alimentación es similar al de escarabajos estercoleros, los cuales se alimentan del líquido en suspensión del sustrato que colonizan (Holter y Scholtz, 2007). Esto, sumado al posible origen filogenético común entre escarabajos detritívoros y escarabajos estercoleros, sugiere una posible relación de estas especies de aphodiinos con estiércol animal (Scholtz et al., 2009), por lo que no se descarta la hipótesis de que podrían desarrollar parte de su ciclo de vida asociadas a estiércol animal.

Es necesario precisar que la clasificación en el uso de las fecas, utilizada en la presente revisión para $F$. costulatus, $H$. punctatissimus, T. elguetai y T. meridionallis, fue realizada básicamente por análisis morfológico de sus patas traseras y delanteras, además de la posición taxonómica en la que fueron clasificados, debido a que no existen estudios que evalúen el comportamiento que poseen estos escarabajos. En contraposición a lo anterior, si es conocido el comportamiento de las otras especies antes mencionadas, lo que permite clasificarlos con seguridad según su comportamiento (Ovalle y Solervicens, 1980; Klemperer, 1983; Howden y Peck, 1987; Conti et al., 1994; Ocampo y Molano, 2011; González, 2013).

\section{ESTUDIOS ECOLÓGICOS SOBRE ESCARABAJOS ESTERCOLEROS EN CHILE}

El primer aporte biológico sobre especies estercoleras fue publicado a principios del siglo XX (Joseph, 1929), correspondiendo a una descripción del com- 
portamiento de Pinotus torulosus (Eschscholtz, 1822) ( $=$ H. torulosus) en época estival, en los alrededores de la ciudad de Temuco, en el sur de Chile. A pesar de ser un trabajo descriptivo, se entregan las primeras observaciones sobre el movimiento y la actividad de esta especie entre el bosque nativo y la pradera, así como su preferencia por estiércol de bovino y equino. Adicionalmente, el autor describe por primera vez las crotovinas de esta especie. Una crotovina (del ruso крото́вина = krotovina en inglés) es un túnel o madriguera de origen animal el cual es rellenado con materia orgánica y/o suelo (Chesworth, 2008). En H. torulosus, corresponde a una bola de estiércol construida al final de un túnel al interior del suelo en donde se desarrolla la fase larval de este insecto (Klemperer, 1983). La misma forma esférica se encuentra en $M$. villosa (Ovalle y Solervicens, 1980), sin embargo, para F. variolosus y T. patagonicus, esta crotovina es de forma cilíndrica (Howden y Peck, 1987; Conti et al., 1994). Más de 50 años después, se realizó el primer estudio sobre la construcción de crotovinas y la arquitectura de nidos por acción de $H$. torulosus (Klemperer, 1983). Algunas características de nidificación han sido descritas también para M. villosa (Ovalle y Solervicens, 1980) y T. patagonicus (Howden y Peck, 1987; Conti et al., 1994). Ambas especies, poseen comportamiento telecóprido, pero el enterramiento de estiércol es más superficial, en comparación con las galerías realizadas por organismos paracópridos, las cuales pueden eventualmente superar los 2 metros de profundidad (Joseph, 1929). M. villosa construye una esfera desde una porción de estiércol, preferentemente de vacuno o equino (aunque anecdóticamente se le asocia también a fecas de perro (Canis lupus familiaris (Linnaeus, 1758)) y burro (Equus africanus asinus (Linnaeus, 1758))), para posteriormente transportarla horizontalmente alrededor de $65 \mathrm{~cm}$ desde el origen (pero hasta 2,5 m) para luego enterrarla entre 10 y $45 \mathrm{~cm}$ al interior del suelo, concentrando sus nidos a una profundidad entre 21 y $35 \mathrm{~cm}$ (Ovalle y Solervicens, 1980). A pesar de poseer comportamiento telecóprido, T. patagonicus no construye una esfera de estiércol. Esta especie utiliza estiércol de roedores (v.g., Dolichotis patagonum (Zimmermann, 1780)) (Zunino, 1984), guanaco (Lama guanicoe (Müller, 1776)), ñandú (Pterocnemia pennata (D’Orbigny, 1834)), zorro (Lycalopex griseus (Gray, 1837)) y recientemente estiércol de ovino (Ovis orientalis (Linnaeus, 1758)) y equino (Equus caballus (Linnaeus, 1758)) (Conti et al., 1994), el cual toma con sus patas delanteras para luego desplazarse "retrocediendo" hacia su nido, recorriendo alrededor de 1,5 m desde el origen del estiércol, enterrándolo entre 2 y $10 \mathrm{~cm}$ al interior del suelo (Conti et al., 1994). No obstante, el estudio de Howden y Peck, (1987), muestra diferencias en la profundidad y características del nido (v.g., diámetro del túnel del nido, ángulo del túnel) las cuales cambian cuando poblaciones de esta especie fueron muestreadas a menor latitud, encontrándose las crotovinas entre 10 y $35 \mathrm{~cm}$ al interior del suelo (Howden y Peck, 1987). Estas diferencias podrían deberse a cambios en el comportamiento del insecto, relacionados a variaciones climáticas en el sentido latitudinal, concentrándose las cámaras de crianza en profundidades en que se presenta el óptimo térmico del suelo $\left(14^{\circ} \mathrm{C}\right.$ como promedio), que en el caso de Tierra del Fuego se encuentra entre 5 y $10 \mathrm{~cm}$ de profundidad (Conti et al., 1994). A menores latitudes, con mayores temperaturas a nivel de la superficie del suelo, este óptimo se encontraría a mayor profundidad lo que llevaría a estos organismos a construir sus nidos en horizontes de suelo más profundos (Conti et al., 1994). Sin embargo, no existen estudios sobre metabolismo en $T$. patagonicus que permitan establecer rangos de temperatura óptimos para el desarrollo de esta especie. Luego de colectar una porción de estiércol, T. patagonicus se desplaza en línea recta hacia el lugar donde éste será enterrado (Conti et al., 1994). Al poseer actividad diurna, es posible que este insecto utilice la posición del sol en el cielo para alejarse en línea recta desde la pila de estiércol, como ha sido demostrado para Scarabaeus lamarcki (MacLeay, 1821) (Dacke et al., 2014). Interesantes avances han ocurrido durante los últimos 10 años en esta área, pues se ha demostrado que ciertos escarabajos estercoleros nocturnos utilizan la luz de la luna, las estrellas e incluso la vía láctea para su orientación (Dacke et al., 2013). Basado en lo anterior, existe la posibilidad que este mecanismo de orientación juegue un rol importante en el establecimiento de estercoleros con comportamiento crepuscular tales como $M$. villosa (Ovalle y Solervicens, 1980). Esto debiese ser considerado al momento de estudiar patrones de dispersión y establecimiento en especies telecópridas, especialmente si se consideran estrategias que apunten hacia aumentar su actividad en agroecosistemas ganaderos.

La distribución de las especies nativas en Chile se presenta en distintas zonas a lo largo del país. Esto se debe principalmente a la adaptación que cada una de estas especies tiene con la presencia de estiércol de mamíferos nativos, el tipo de vegetación y las condiciones climáticas. Estos factores han modelado la evolución de estos insectos en distintas partes del mundo (Nichols et al., 2009; Scholtz et al., 2009; Simmons y Ridsdill-Smith, 2011). Sin embargo, es importante señalar que existen factores que afectan su distribución en escalas de tiempo mucho menores, como lo son la deforestación y fragmentación de hábitat (Nichols et al., 2007), la construcción de caminos (Hosaka et al., 2014), el uso de plaguicidas (Davis et al., 2004) y el cambio de uso de suelo por acción antrópica (Barragán et al., 2011). Estos factores podrían explicar la actual distribución de especies coprófagas presentes en Chile, así como la baja actividad de estos insectos en procesos ecosistémicos en sistemas ganaderos intensivos de la zona centro-sur de Chile. 
Desde un punto de vista histórico, Gutiérrez (1947) comenta por primera vez la distribución geográfica que presentan algunos de estos escarabajos, tanto en Chile como en Argentina. Una exhaustiva recopilación de la distribución latitudinal de las especies nativas en Chile ha sido compilada por Smith (2015). Adicionalmente, a esta variación latitudinal natural que presenta Chile dada su diversa geografía, existen diferencias en la preferencia de hábitat por parte de estos insectos. Hasta la fecha, existen solo dos estudios que evalúan la población de escarabajos estercoleros en dos hábitats contrastantes; pradera natural y bosque nativo, en el sur de Chile (González, 2010; 2013). En el primer estudio de González, (2010) se comparó la abundancia de $H$. torulosus en ambos hábitats en la Región de los Ríos, encontrando mayor cantidad de estercoleros al interior del bosque nativo, en comparación a la pradera. Sin embargo, en este estudio solo se evaluó la época otoñal, por lo que no es posible concluir la preferencia de hábitat de este insecto durante el resto del año. No obstante de lo anterior, en ciertas especies estercoleras existe una marcada preferencia entre bosque y pradera, encontrándose especies que comparten ambos hábitats, mientras que otras son exclusivas a uno de éstos (Escobar et al., 2007; Horgan, 2007). En el segundo estudio de González, (2013) se compararon estos mismos hábitats, pero en distintas zonas de la Región de Aysén. Estas mediciones se realizaron durante un año completo (temporada 2011-2012), encontrando distintos resultados para cada zona. En las zonas con menor intensidad en el uso de insumos agrícolas, los escarabajos se concentraron sobre praderas naturales, en cambio, en zonas con mayor intensidad en el uso de estos productos, éstos se concentraron al interior del bosque nativo. Esto sugiere un efecto del manejo ganadero sobre la abundancia de estos insectos, lo cual ha sido demostrado para $F$. variolosus en manejos como la aplicación de distintos antiparasitarios (ivermectina, moxidectina y fenbendazol); así como en el uso de rastras de goma (rastra con neumáticos para la disgregación de la pila de estiércol), los cuales afectaron el desarrollo larval y la construcción de túneles, respectivamente (De la Vega et al., 2013; González, 2013). Debido a que los estudios mencionados anteriormente se realizaron en una zona acotada de la Región de Aysén, no es posible atribuir la variación espacial en la preferencia de hábitat de estos escarabajos con el manejo ganadero, lo cual no obstante, ha sido demostrado para otras especies estercoleras (Numa et al., 2012; Wall y Beynon, 2012). Un estudio que considere la variabilidad espacial de los manejos ganaderos en la Región de Aysén (v.g., uso de antiparasitarios, uso de rastras de goma), así como la distribución y preferencia de hábitat de $F$. variolosus, podría contribuir con un mejor entendimiento de las dinámicas espaciales de este insecto en la región.
Por otro lado, existen variaciones altitudinales en la distribución de estos insectos, las cuales fueron descritas por primera vez en Chile para H. torulosus, F. variolosus y A. gracilis, quienes se distribuyeron hasta 1100, 1200 y 700 msnm, respectivamente (Gutiérrez, 1949). Sin embargo, Parra et al. (2005) sugieren que H. torulosus solo se encuentra hasta los $500 \mathrm{msnm}$, mientras que $F$. variolosus entre los 350 y los $1500 \mathrm{msnm}$, ambos muestreados en la Región de la Araucanía. En la Región de Aysén, la presencia de $F$. variolosus varía entre 3 y 725 msnm (González, 2013). Estas variaciones en la distribución altitudinal podrían estar dadas por variaciones microclimáticas y vegetacionales, provocadas por un aumento en altitud, lo cual ha sido descrito para otras especies estercoleras en Perú (Larsen, 2012).

Dada la especificidad que muestran estos insectos a ciertas condiciones microclimáticas (v.g., luminosidad, temperatura, humedad), es que recientemente se han utilizado para entender procesos de deforestación y fragmentación de ecosistemas boscosos y como éstos afectan la diversidad local de mamíferos (Nichols et al., 2009; Nichols y Gardner, 2011). Esta relación con mamíferos nativos sugiere otro punto de vista para entender la distribución y las diferencias de hábitat que presentan estas especies en base a la presencia de estos mamíferos en términos evolutivos (Scholtz et al., 2009; Philips, 2011). Estos factores, tanto bióticos (presencia de mamíferos, tipo de vegetación) como abióticos (clima), han sido mencionados anteriormente como los causantes en la distribución actual de T. patagonicus en la estepa patagónica (Zunino, 1984) y en Tierra del Fuego (Conti et al., 1994), tras millones de años de evolución. Una aproximación a este marco conceptual, pero a menor escala espacial, se realizó recientemente utilizando distintos tipos de estiércol de mamíferos, tanto nativos como exóticos, sobre la atracción de individuos de F. variolosus (González, 2013). En este estudio, $F$. variolosus fue evaluado frente a estiércol de vacuno, caballo, huemul (Hippocamelus bisulcus (Molina, 1782)), guanaco (Lama guanicoe (Müller, 1776)) y puma (Puma concolor (Molina, 1782)), mostrando éste último la menor cantidad de escarabajos atraídos y siendo significativamente distinto a los demás. Sin embargo, no existieron diferencias en la atracción hacia estiércol de bovino y de huemul. Es más, la gran cantidad de individuos atraídos hacia estiércol de huemul, sugiere que este insecto pudo haber utilizado este recurso, anterior a la colonización de animales vacunos en la región, durante el siglo XIX. Basado en registros zoo-arqueológicos, se sugiere que la distribución del huemul hace 500 años se concentraba principalmente en praderas del valle central de Chile (Flueck y SmithFlueck, 2012). Posteriormente, debido a múltiples factores socio-ecológicos, estos cérvidos se desplazaron hacia zonas montañosas más protegidas, cambiando la condición de pradera natural por la de bosque na- 
tivo (Flueck y Smith-Flueck, 2012). Esta migración del huemul pudo eventualmente promover una condición "generalista" en la preferencia de hábitat de F. variolosus, al encontrarse con abundante alimento en zonas boscosas, a medida que el huemul cambiaba las praderas naturales por el bosque nativo. Esta idea ha sido recientemente demostrada en Japón, donde al aumentar la abundancia de ciervos (Cervus nippon (Temminck, 1838)), aumentó la cantidad de escarabajos estercoleros paracópridos (Koike et al., 2014). Sin embargo, más estudios son necesarios para poder atribuir las diferencias en abundancia entre bosque nativo y pradera encontradas por (González, 2013) a condiciones evolutivas o a manejos ganaderos actualmente realizados en la zona o a ambos factores actuando conjuntamente. Cabe señalar, que lo anteriormente propuesto es solo una aproximación que intenta explicar las diferencias en preferencia de hábitat encontradas por (González, 2013), ya que aunque éstos insectos sean atraídos por cierto tipo de alimento, no necesariamente existe una evolución entre éstos y el tipo de cebo utilizado. Por ejemplo, se ha utilizado calamar descompuesto para colectar escarabajos estercoleros en Nueva Zelanda (v.g., Jones et al., 2012), y por supuesto, esto no significa que existe una evolución entre calamares y escarabajos estercoleros. Es necesario evaluar el uso de distintos de tipos de estiércol, sobre la capacidad de construcción de nidos y la supervivencia de larvas de F. variolosus, para avanzar en el entendimiento de esta especie con su entorno. Hasta el momento, solo se han encontrado crotovinas de este insecto hechas con estiércol bovino.

\section{LOS ESCARABAJOS ESTERCOLEROS NATIVOS Y SU ROL EN LA SUSTENTABILIDAD DEL POTENCIAL GANADERO DEL SUR DE CHILE}

En el sur de Chile, desde Los Ángeles $\left(37^{\circ} 28^{\prime} \mathrm{S}\right.$;

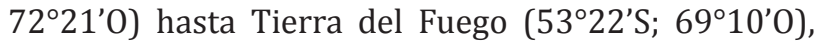
se concentran distintas actividades ganaderas, destacándose la producción de leche y carne bovina, además de carne y fibra ovina. Dada la amplitud geográfica que presentan estas actividades ganaderas en el país, así como la importancia social y cultural que representan, se hace imprescindible mantener su productividad y al mismo tiempo, promover la sustentabilidad de dichos agroecosistemas, especialmente en el actual escenario de pérdida de biodiversidad y cambio climático (Steffen, 2010). Una de las mayores fuentes de gases de efecto invernadero que contribuyen al cambio climático, provienen de la agricultura y la producción de alimentos (Smith et al., 2008; Bellarby et al., 2013). Se estima que entre el 18 y 51\% de éstos provienen de la producción animal (Steinfeld et al., 2006; Goodland, 2014). Recientemente, se demostró que los escarabajos estercoleros son capaces de reducir la cantidad de metano liberado a la atmósfera desde las pilas de estiércol depositadas por bovinos (Penttilä et al., 2013; Iwasa et al., 2015). Este es un interesante hallazgo que podría contribuir a reducir la cantidad de gases de efecto invernadero liberados a la atmosfera en la producción ganadera.

Por otra parte, al incorporar el estiércol al interior del suelo, se generan una serie de beneficios, entre los que destacan el aumento en el contenido de nutrientes (Bertone, 2004; Lastro, 2006; González, 2013), cambios en la conductividad hidráulica del suelo (Brown et al., 2010), aumento en la conductividad de aire del suelo (Bang et al., 2005) y disminución de las emisiones de nitrógeno desde el suelo hacia la atmosfera, a través de un aumento en la fijación del mismo (Yokoyama et al., 1991). Esto contribuye con un aumento del rendimiento en las praderas y la calidad del forraje producido (Bang et al., 2005), promoviendo una mejor utilización de la misma, al remover el estiércol de la superficie del suelo, permitiendo un crecimiento uniforme de ésta (Waterhouse, 1974). Adicionalmente, tras enterrar el estiércol, estos insectos contribuyen con el control de moscas (v.g., Haematobia sp., Musca sp.) (Edwards y Aschenborn, 1987; Ripa et al., 1995; Nichols et al., 2008) y control de parásitos intestinales (v.g., Trichostrongylidae) (Fincher, 1975), los cuales afectan al ganado bovino. Esto es relevante, tanto desde el punto de vista del bienestar animal como desde la salud ambiental, al disminuir el uso de productos veterinarios (Losey y Vaughan, 2006), los cuales recientemente han mostrado efectos no deseados sobre otras comunidades de insectos que colonizan el estiércol (Wall y Beynon, 2012; Sutton et al., 2014). Lo anteriormente mencionado, se traduce para el agricultor en una eventual reducción de sus costos de producción (Losey y Vaughan, 2006), y desde un punto de vista ambiental, contribuye con la conservación de la biodiversidad local, aumentando la resiliencia de estos sistemas ganaderos (Tilman et al., 2006). No obstante, hasta el momento, no existe un estudio que relacione directamente la actividad de escarabajos estercoleros con un aumento en la diversidad animal al interior o sobre el suelo. Sin embargo, su rol en la dispersión y establecimiento de semillas, promoviendo un aumento de la diversidad vegetal es reconocido para distintos ecosistemas alrededor del mundo (v.g., Slade et al., 2007; Verdú et al., 2009; Kunz y Krell, 2011; Feer et al., 2012).

Basado en el actual conocimiento que existe sobre la relación de especies estercoleras nativas y estiércol de animales introducidos, es posible señalar que $H$. torulosus y $F$. variolosus podrían generar los beneficios mencionados anteriormente en la producción bovina, mientras que $T$. patagonicus podría contribuir con la producción ovina, especialmente en sistemas ovinos de ambientes patagónicos extremos en el sur de Chile, en donde no existe aplicación de fertilizantes a las praderas, debido a la gran extensión de los predios. Re- 
cientemente se ha demostrado el efecto de $F$ variolosus sobre la desaparición y entierro de estiércol de bovino, quien al sexto día después de colonizar el estiércol, logró remover más del 60\% de éste (González, 2013), lo que podría contribuir a la reducción de poblaciones de mosca de los cuernos (Haematobia irritans (Linnaeus, 1758)). No obstante, el estudio anterior se realizó bajo condiciones de invernadero, por lo que estudios futuros debiesen evaluar el efecto de estos organismos coprófagos en condiciones naturales. En este sentido, investigar el efecto de la densidad del ganado por unidad de superficie y su efecto en la cantidad y distribución de estiércol presente en cada potrero sobre la abundancia y riqueza de especies estercoleras es fundamental para diseñar programas de manejo ganadero que apunten a promover la actividad de estos insectos. A la fecha, no existe ningún artículo que evalúe estrategias de manejo ganadero per se para promover la actividad de organismos estercoleros nativos, por lo que avances en esta área contribuirán enormemente al desarrollo de la conservación de la comunidad estercolera nativa. Adicionalmente, la fauna asociada al estiércol, distinta de coprófagos, debe ser considerada sobre las dinámicas de parásitos. Por ejemplo, ácaros foréticos asociados a escarabajos estercoleros han sido descritos como predadores de nematodos y larvas de moscas presentes en las fecas (Niogret et al., 2006), por lo que su abundancia y diversidad podrían contribuir con el control de parásitos que afectan al ganado bovino en el sur de Chile. La única especie descrita actualmente, Rhizoglyphus frickorum (Nesbitt, 1988) ha sido asociada a F. variolosus (Nesbitt, 1988), pero su potencial rol como controlador biológico es aún desconocido.

Por otro lado, se encontró que tras enterrar el estiércol, $F$ v variolosus aumentó en $4 \%$ el contenido de fósforo en el suelo, disminuyendo al mismo tiempo la cantidad de aluminio extractable en 19\% (González, 2013). Especial relevancia tiene este descubrimiento, sobre todo considerando que parte de la producción ganadera en el sur de Chile se realiza sobre suelos de origen volcánico (Andisol), los cuales poseen restricciones en la disponibilidad de fósforo y problemas por toxicidad de aluminio a las plantas, lo cual restringe el crecimiento vegetal (Dahlgren et al., 2004). A pesar de lo anterior, no existen en Chile estudios que evalúen los efectos de las comunidades coprófagas sobre los diferentes servicios ecosistémicos recopilados por Nichols et al. (2008) en agroecosistemas ganaderos. En Japón, tras evaluar los efectos de los estercoleros sobre el contenido de nutrientes y el rendimiento en una pradera, se observó un aumento de éstos tras liberar 40 escarabajos en una parcela experimental (Yamada et al., 2007). Sin embargo, este aumento solo se encontró al inicio del experimento, y la relación entre estas variables de respuesta y la presencia de escarabajos estercoleros no fue significativa durante el resto de los 2 años en que se evaluó el experimento. Considerando que la mayoría de los estudios relacionados a entender los efectos de estos escarabajos sobre el contenido de nutrientes y el rendimiento en praderas se han realizado bajo condiciones de laboratorio y/o invernadero, el efecto en condiciones de campo podría estar diluido por otras variables ambientales que también contribuyen con la degradación del estiércol. Adicionalmente, el tipo de alimento que consume el ganado bovino podría afectar las dinámicas de incorporación de estiércol. Recientemente, al estudiar la relación entre biodiversidad coprófaga y funcionamiento de un agroecosistema ganadero, se encontró que un aumento en el contenido de nutrientes del suelo por entierro de estiércol varió en función de la alimentación recibida por el ganado estudiado (Yoshihara y Sato, 2015). Estiércol proveniente de ganado alimentado con pasto fue incorporado en mayor cantidad al compararlo con estiércol proveniente de animales alimentados con grano. El trabajo citado anteriormente sugiere que el tipo de alimentación puede jugar un rol importante en la remoción de estiércol bovino, aun cuando las comunidades coprófagas estén adaptadas a él.

\section{DESAFÍOS FUTUROS}

Este trabajo ha recopilado la información existente en Chile sobre escarabajos estercoleros nativos, con énfasis en el estudio de su comportamiento, biología y ecología. En Chile, a pesar de presentar una variada diversidad de especies, la investigación científica se ha concentrado principalmente en su descripción taxonómica, siendo muy pocos los trabajos que aportan información sobre su biología y/o ecología. Ya que estos insectos son útiles bioindicadores de la calidad ambiental, dada su sensibilidad a perturbaciones antrópicas (Scholtz et al., 2009; Nichols y Gardner, 2011), su conservación pasa necesariamente por la conservación de sus hábitats, lo cual sugiere la posibilidad de utilizar una aproximación similar a la del concepto "Umbrella species" en donde una especie "carismática" es conservada, promoviendo la conservación de muchas otras especies que comparten su mismo rango de distribución (Sergio et al., 2006; Breckheimer et al., 2014). Bajo esta concepción, pareciese que los escarabajos estercoleros nativos pueden contribuir de manera significativa a la producción animal y la conservación de sus ecosistemas nativos, promoviendo de manera indirecta un aumento en el bienestar del ser humano, ya que recientemente, se ha planteado la directa relación que existe entre la biodiversidad y el bienestar humano (Fuller et al., 2007; Dallimer et al., 2012). Sin embargo, para reconocer a los escarabajos estercoleros nativos como agentes promotores de la sustentabilidad ganadera del sur de Chile y la conservación de ecosistemas nativos, son necesarios más estudios que evalúen los efectos de estos insectos a nivel de campo, poniendo énfasis en 
manejos que pudiesen afectar o favorecer su abundancia y actividad. Se reconoce que tanto factores biogeográficos como ecológicos afectan la abundancia y diversidad de estos insectos (Barragán et al., 2014), así como el manejo agrícola que presenta cada predio (Numa et al., 2012), por lo que la sola disminución en la aplicación de agroquímicos y/o productos antiparasitarios no necesariamente restaurará la comunidad coprófaga original presente en cierto lugar. A pesar de la importancia de estudios ecológicos aplicados en agricultura sustentable (Robertson y Swinton, 2005; Brussaard et al., 2010), escasos son los trabajos que evalúen el efecto de tipos de manejos aplicados a la ganadería sobre la coprofauna existente localmente. No obstante, en los Andes Colombianos, se determinó que la presencia de sistemas silvopastoriles aumentaron la abundancia estercolera, acelerando la tasa de remoción de estiércol, lo que en consecuencia disminuyó la cantidad de larvas de mosca que afectan al ganado bovino (Giraldo et al., 2011). Actualmente en Chile, tanto $H$. torulosus como $F$. variolosus presentan una relación con formaciones boscosas nativas, lo que sugiere su importancia en el desarrollo de estas especies. La posible implementación de sistemas silvopastoriles con especies nativas podría contribuir con la restauración de la actividad de estos y otros organismos, promoviendo la conservación de la biodiversidad local. Una alternativa para incrementar las interacciones entre praderas y bosques nativos, podría ser la inclusión de cercos vivos en los bordes de los predios, así como al interior de los mismos. Sin embargo, la implementación de estos cordones de vegetación nativa requiere de un estudio exhaustivo de las interacciones tróficas que ellos promueven, ya que al potenciar la comunidad de enemigos naturales mediante este manejo vegetal (Landis et al., 2000; Wratten et al., 2012), éstas podrían afectar las poblaciones de escarabajos estercoleros mediante predación. Sin embargo, al sur de México la presencia de cercos vivos presentó similar diversidad de escarabajos al ser comparados con remanentes de bosque nativo, siendo ésta mayor al compararla con sus praderas naturales asociadas (Arellano et $a l ., 2008$ ). Esto sugiere que el potencial efecto de predación por parte de enemigos naturales debiese ser analizado considerando las características locales en cada agroecosistema, ya que en esta experiencia Mexicana, el cerco vivo estaría proveyendo refugio a estas especies estercoleras (v.g., ante variaciones microclimáticas y/o ataque de aves insectívoras), como ocurre con otros insectos cuando estas formaciones vegetales son incluidas en sistemas agrícolas (Landis et al., 2000). Es necesario precisar que esta manipulación de hábitat, a través de cambios en la vegetación, no solo podría contribuir con la conservación de la comunidad estercolera presente en Chile, sino que también con otras especies animales al promover distintos servicios ecosistémicos, tales como regulación hídrica, polinización, control bio- lógico, desarrollo rural y estética paisajística (Wratten et al., 2012). La producción de estos servicios ecológicos en sistemas ganaderos podría ser la base para estimular la sustentabilidad agrícola en Chile.

Finalmente, la escasez de publicaciones científicas en Chile durante los últimos 100 años en torno a la relación de estos insectos con su entorno, mantiene esta área de la entomología en Chile aún en un estado muy precario. Sin embargo, existe una gran oportunidad de desarrollar investigación dentro de un marco de mejora en la sustentabilidad de los sistemas ganaderos y agrícolas en Chile. El avance de la investigación científica sobre el rol de las comunidades coprófagas en la regulación de diversos servicios ecosistémicos producidos en sistemas ganaderos del sur de Chile, sugiere un prometedor futuro en la re-unión de la ecología con la producción agrícola.

\section{AGRADECIMIENTOS}

Se agradece a Mario Elgueta, del Museo Nacional de Historia Natural (Santiago, Chile), por su invaluable contribución a una versión preliminar de este manuscrito. A dos revisores anónimos, se agradecen sus comentarios y atingentes sugerencias que mejoraron la versión final de este manuscrito.

\section{REFERENCIAS}

Arellano, L., León-Cortés, J.L., Halffter, G., 2008. Response of dung beetle assemblages to landscape structure in remnant natural and modified habitats in southern Mexico. Insect Conservation and Diversity 1(4), 253-262. http://dx.doi.org/10.1111/j.1752-4598.2008.00033.x

Bang, H.S., Lee, J., Kwon, O.S., Na, Y.E., Jang, Y.S., Kim, W.H., 2005. Effects of paracoprid dung beetles (Coleoptera: Scarabaeidae) on the growth of pasture herbage and on the underlying soil. Applied Soil Ecology 29, 165-171. http://dx.doi.org/10.1016/j.apsoil.2004.11.001

Barragán, F., Moreno, C.E., Escobar, F., Bueno-Villegas, J., Halffter, G., 2014. The impact of grazing on dung beetle diversity depends on both biogeographical and ecological context. Journal of Biogeography 41, 1991-2002. http:// dx.doi.org/10.1111/jbi.12351

Barragán, F., Moreno, C.E., Escobar, F., Halffter, G., Navarrete, D., 2011. Negative impacts of human land use on dung beetle functional diversity. PloS One 6(3), e17976. http://dx.doi.org/10.1371/journal.pone.0017976

Bellarby, J., Tirado, R., Leip, A., Weiss, F., Lesschen, J.P., Smith, P., 2013. Livestock greenhouse gas emissions and mitigation potential in Europe. Global Change Biology 19(1), 3-18. http://dx.doi.org/10.1111/j.13652486.2012.02786.x

Bertone, M., 2004. Dung beetles (Coleoptera: Scarabaeidae and Geotrupidae) of North Carolina cattle pastures and their implications for pasture improvement. Tesis Magíster Entomología, North Carolina State University. 159p.

Bornemissza, F., 1976. The Australian dung beetle proyect. 
Australian Meat Research Committee Review 30, 1-30.

Breckheimer, I., Haddad, N.M., Morris, W.F., Trainor, A.M., Fields, W.R., Jobe, R.T., Hudgens, B.R., Moody, A., Walters, J.R., 2014. Defining and evaluating the Umbrella Species concept for conserving and restoring landscape connectivity. Conservation Biology 28(6), 1584-1593. http:// dx.doi.org/10.1111/cobi.12362

Brown, J., Scholtz, C.H., Janeau, J.-L., Grellier, S., Podwojewski, P., 2010. Dung beetles (Coleoptera: Scarabaeidae) can improve soil hydrological properties. Applied Soil Ecology 46(1), 9-16. http://dx.doi.org/10.1016/j.apsoil.2010.05.010

Brussaard, L., Caron, P., Campbell, B., Lipper, L., Mainka, S., Rabbinge, R., Babin, D., Pulleman, M., 2010. Reconciling biodiversity conservation and food security: scientific challenges for a new agriculture. Current Opinion in Environmental Sustainability 2(1-2), 34-42. http://dx.doi. org/10.1016/j.cosust.2010.03.007

Campos, L., Peña, L., 1973. Los insectos de Isla de Pascua. Revista Chilena de Entomología 7, 217-229.

Chesworth, W., 2008. Encyclopedia of Soil Science. Springer, Dordrecht, The Netherlands.

Conti, E., Costa, G., Petralia, A., Sobral, A., 1994. Eco-ethological research on the Antarctic and Subantarctic fauna. 4. Biology of Taurocerastes patagonicus Phil. (Coleoptera, Geotrupidae). Anales del Instituto de la Patagonia: Las Ciencias Naturales 22, 69-78.

Dacke, M., Baird, E., Byrne, M., Scholtz, C.H., Warrant, E.J., 2013. Dung beetles use the Milky Way for orientation. Current Biology 23(4), 298-300. http://dx.doi.org/10.1016/j. cub.2012.12.034

Dacke, M., el Jundi, B., Smolka, J., Byrne, M., Baird, E., 2014. The role of the sun in the celestial compass of dung beetles. Philosophical Transactions of the Royal Society B 369, 1-7. http://dx.doi.org/10.1098/rstb.2013.0036

Dahlgren, R.A., Saigusa, M., Ugolini, F.C., 2004. The nature, properties, and management of volcanic soils. Advances in Agronomy 82, 113-182.

Dallimer, M., Irvine, K.N., Skinner, A.M.J., Davies, Z.G., Rouquette, J.R., Maltby, L.L., Warren, P.H., Armsworth, P.R., Gaston, K.J., 2012. Biodiversity and the feel-good factor: Understanding associations between self-reported human well-being and species richness. BioScience 62(1), 47-55. http://dx.doi.org/10.1525/bio.2012.62.1.9

Davis, A., Scholtz, C., Dooley, P., Bham, N., Kryger, U., 2004. Scarabaeine dung beetles as indicators of biodiversity, habitat transformation and pest control chemicals in agro-ecosystems. South African Journal of Science 100, 415-424.

De la Vega, C., Elizalde, F., Gonzalez, M., 2013. Efectos del uso de rastras de goma como disgregadores de bosta sobre el escarabajo estercolero Frickius variolosus Germain, 1897 en la Región de Aysén. XXXVIII Congreso Anual de la Sociedad Chilena de Producción Animal A. G. (SOCHIPA). Instituto de Investigaciones Agropecuarias, 23 al 25 de Octubre de 2013, Frutillar, Chile, pp. 177-178.

Edwards, P., Aschenborn, H., 1987. Patterns of nesting and dung burial in Onitis dung beetles: Implications for pasture productivity and fly control. Journal of Applied Ecology 24, 837-851. http://dx.doi.org/10.2307/2403984

Escobar, F., Halffter, G., Arellano, L., 2007. From forest to pas- ture: An evaluation of the influence of environment and biogeography on the structure of dung beetle (Scarabaeinae) assemblages along three altitudinal gradients in the Neotropical region. Ecography 30, 193-208. http://dx.doi.org/10.1111/j.2007.0906-7590.04818.x

Feer, F., Ponge, J.-F., Jouard, S., Gomez, D., 2012. Monkey and dung beetle activities influence soil seed bank structure. Ecological Research 28(1), 93-102. http://dx.doi. org/10.1007/s11284-012-1006-9

Fincher, G., 1975. Effects of dung beetle activity on number of nematode parasites acquired by grazing cattle. Journal of Parasitology 61, 759-762. http://dx.doi. org/10.2307/3279480

Fincher, G.T., Stewart, T.B., Hunter III, J.S. 1983., The 1981 distribution of Onthophagus gazella Fabricius from releases in Texas and Onthophagus taurus Schreber from an unknown release in Florida (Coleoptera: Scarabaeidae). The Coleopterists Bulletin 37(2), 159-163. http://www. jstor.org/stable/4008007

Flueck, W., Smith-Flueck, J.A., 2012. Huemul heresies: Beliefs in search of supporting data. 1. Historical and zooarcheological considerations. Animal Production Science 52(8), 685-693. http://dx.doi.org.ezproxy.lincoln. ac.nz/10.1071/AN11344\#sthash.U1T8fs0V.dpuf

Fuller, R.A., Irvine, K.N., Devine-Wright, P., Warren, P.H., Gaston, K.J., 2007. Psychological benefits of greenspace increase with biodiversity. Biology Letters 3(4), 390-394. http://dx.doi.org/10.1098/rsbl.2007.0149

Giller, P., Doube, B., 1989. Experimental analysis of inter and intraspecific competition in dung beetle communities. Journal of Animal Ecology 58, 129-142. http://dx.doi. org/10.2307/4990

Giraldo, C., Escobar, F., Chará, J.D., Calle, Z., 2011. The adoption of silvopastoral systems promotes the recovery of ecological processes regulated by dung beetles in the Colombian Andes. Insect Conservation and Diversity 4(2), 115-122. http://dx.doi.org/10.1111/j.17524598.2010.00112.x

González, M., 2010. Presencia otoñal de escarabajos estercoleros nativos paracópridos (Scarabaeidae: Scarabaeinae) en renovales de bosque nativo y praderas naturales asociadas. Tesis Agronomía, Universidad Austral de Chile. 82p.

González, M., 2013. Ecología del escarabajo estercolero nativo paracóprido Frickius variolosus Germain, 1897 (Coleoptera, Geotrupidae), en la Región de Aysén. Tesis Magíster Ciencias del Suelo, Universidad Austral de Chile. 73p.

Goodland, R., 2014. A fresh look at livestock greenhouse gas emissions and mitigation potential in Europe. Global Change Biology 20(7), 2042-2044. http://dx.doi. org/10.1111/gcb.12454

Gutiérrez, R., 1947. Escarabajos comunes a Chile y la Argentina. Revista de la Sociedad Entomológica Argentina 13, 309-314.

Gutiérrez, R., 1949. Notas sobre los Scarabaeidae neotrópicos. Anales de la Sociedad Científica Argentina 148(6), 9-35.

Halffter, G., Edmonds, W.D., 1982. The nesting behavior of dung beetles (Scarabaeinae). An ecological and evolutive approach. Instituto de Ecología, México.

Hanski, I., Cambefort, Y., 1991. Dung Beetle Ecology. Prince- 
ton University Press, New Jersey, United States.

Holter, P., Scholtz, C.H., 2007. What do dung beetles eat? Ecological Entomology 32(6), 690-697. http://dx.doi. org/10.1111/j.1365-2311.2007.00915.x

Horgan, F., 2007. Dung beetles in pasture landscapes of Central America: Proliferation and synanthropogenic species and decline of forest specialists. Biodiversity and Conservation. 16, 2149-2165. http://dx.doi. org/10.1007/s10531-006-9145-3

Horgan, F., Fuentes, R., 2005. Asymmetrical competition between Neotropical dung beetles and its consequences for assemblage structure. Ecological Entomology 30, 182-193. http://dx.doi.org/10.1111/j.03076946.2005.00673.x

Hosaka, T., Niino, M., Kon, M., Ochi, T., Yamada, T., Fletcher, C., Okuda, T., 2014. Effects of logging road networks on the ecological functions of dung beetles in peninsular Malaysia. Forest Ecology and Management 326, 18-24. http://dx.doi.org/10.1016/j.foreco.2014.04.004

Howden, H.F., Peck, S.B., 1987. Adult habits, larval morphology, and phylogenetic placement of Taurocerastes patagonicus Philippi (Scarabaeidae: Geotrupinae). Canadian Journal of Zoology 65, 329-332. http://dx.doi. org/10.1139/z87-051

Iwasa, M., Moki, Y., Takahashi, J., 2015. Effects of the activity of coprophagous insects on greenhouse gas emissions from cattle dung pats and changes in amounts of nitrogen, carbon, and energy. Environmental Entomology 44(1), 106-113. http://dx.doi.org/10.1093/ee/nvu023

Jones, A., Forgie, S., Scott, D., Beggs, J., 2012. Generalist dung attraction response in a New Zealand dung beetle that evolved with an absence of mammalian herbivores. Ecological Entomology 37(2), 124-133. http://dx.doi. org/10.1111/j.1365-2311.2012.01344.x

Joseph, C., 1929. El Pinotus torulosus Eschsch. Revista Chilena de Historia Natural 33, 31-46.

Klemperer, H., 1983. Brood ball construction by the nonbrooding Coprini Sulcophanaeus carnifex and Dichotomius torulosus (Coleoptera, Scarabaeidae). Ecological Entomology 8, 61-68. http://dx.doi. org/10.1111/j.1365-2311.1983.tb00483.x

Koike, S., Soga, M., Nemoto, Y., Kozakai, C., 2014. How are dung beetle species affected by deer population increases in a cool temperate forest ecosystem ? Journal of Zoology 193, 227-233. http://dx.doi.org/10.1111/jzo.12138

Kunz, B.K., Krell, F.-T., 2011. Habitat differences in dung beetle assemblages in an African savanna-forest ecotone: Implications for secondary seed dispersal. Integrative Zoology 6(2), 81-96. http://dx.doi.org/10.1111/j.17494877.2011.00240.x

Landis, D.A., Wratten, S.D., Gurr, G.M., 2000. Habitat management to conserve natural enemies of arthropod pests in agriculture. Annual Review of Entomology 45, 175-201. http://dx.doi.org/10.1146/annurev.ento.45.1.175

Larsen, T., 2012. Upslope range shifts of Andean dung beetles in response to deforestation: Compounding and confounding effects of microclimatic change. Biotropica 44(1), 82-89. http://dx.doi.org/10.1111/j.17447429.2011.00768.x

Lastro, E., 2006. Dung beetles (Coleoptera: Scarabaeidae and Geotrupidae) in North Carolina pasture ecosystem. Te- sis Magíster Entomología, North Carolina State University. 121p.

Losey, J., Vaughan, M., 2006. The economic value of ecological services provided by insects. BioScience 56, 311-323. http://dx.doi.org/10.1641/00063568(2006)56[311:TEVOES]2.0.CO;2

Miranda, B., 2006. Contribución del escarabajo estercolero africano en la mejoría de la fertilidad del suelo. X Sseminario Manejo y Utilización de Pastos y Forrajes en Sistemas de Producción Animal. Universidad de Zulia, 20-22 de Abril de 2006, Maracaibo, Venezuela, pp. 187200. http://www.avpa.ula.ve/congresos/seminario_ pasto_X/Conferencias/A17-Cesar\%20Behling.pdf

Nesbitt, H., 1988. Three new species of Rhizoglyphine mites from Mexico and Chile. International Journal of Acarology 14(1), 13-18. http://dx.doi. org/10.1080/01647958808683799

Nichols, E., Gardner, T., 2011. Dung beetles as a candidate study taxon in applied biodiversity conservation research, in: Simmons, L., Ridsdill-Smith, J., (Eds.), Ecology and Evolution of Dung Bettles.Wiley-Blackwell, Chichester, pp. 267-291.

Nichols, E., Larsen, T., Spector, S., Davis, A., Escobar, F., Favila, M., Vulinec, K., 2007. Global dung beetle response to tropical forest modification and fragmentation: A quantitative literature review and meta-analysis. Biological Conservation 137, 1-19. http://dx.doi.org/10.1016/j. biocon.2007.01.023

Nichols, E., Spector, S., Louzada, J., Larsen, T., Amezquita, S., Favila, M., 2008. Ecological functions and ecosystem services provided by Scarabaeinae dung beetles. Biological Conservation 141, 1461-1474. http://dx.doi. org/10.1016/j.biocon.2008.04.011

Nichols, E., Gardner, T.A., Peres, C.A., Spector, S., Network, T.S.R., 2009. Co-declining mammals and dung beetles: An impending ecological cascade. Oikos 118(4), 481-487. http://dx.doi.org/10.1111/j.1600-0706.2008.17268.x

Niogret, J., Lumaret, J.-P., Bertrand, M., 2006. Review of the phoretic association between coprophilous insects and macrochelid mites (Acari: Mesostigmata) in France. Elytron 20, 99-121.

Noriega, J., Horgan, F., Larsen, T., Valencia, G., 2010. Records of an invasive dung beetle species, Digitonthophagus gazella (Fabricius, 1787) (Coleoptera: Scarabaeidae), in Peru. Acta Zoológica Mexicana 26(2), 451-456. http:// www.redalyc.org/articulo.oa?id=57517683009

Noriega, J., Moreno, J., Otavo, S., 2011. Quince años del arribo del escarabajo coprófago Digitonthophagus gazella (Fabricius, 1787) (Coleoptera: Scarabaeidae) a Colombia: Proceso de invasión y posibles efectos de su establecimiento. Biota Colombiana 12(2), 35-43. http://www. redalyc.org/articulo.oa?id=49122304004

Numa, C., Verdú, J.R., Rueda, C., Galante, E., 2012. Comparing dung beetle species assemblages between protected areas and adjacent pasturelands in a Mediterranean Savanna landscape. Rangeland Ecology and Management 65(2), 137-143. http://dx.doi.org/10.2111/REMD-10-00050.1

Ocampo, F.C., Molano, F., 2011. Revision and biogeography of the Neotropical dung beetle genus Scybalophagus (Coleoptera: Scarabaeidae). Revista de la Sociedad Entomo- 
lógica Argentina 70(3-4), 231-253. http://www.redalyc. org/articulo.oa?id=322028524008

Ovalle, M., Solervicens, J., 1980. Observaciones sobre la biología de Megathopa villosa Eschscholtz 1822 (Coleoptera: Scarabaeidae: Scarabaeinae). Boletín Museo Nacional de Historia Natural de Chile 37, 235-246.

Parra, L., Mutis, A., Medel, V., Ojeda, N., Rebolledo, R., 2005. Distribución de Dichotomius torulosus (Eschscholtz) (Scarabaeidae) y Frickius variolosus (Germain) (Geotrupidae) en la IX Región de la Araucanía. XXVII Congreso Nacional de Entomología. Universidad Austral de Chile, 23-25 de Noviembre de 2005, Valdivia, Chile.

Penttilä, A., Slade, E.M., Simojoki, A., Riutta, T., Minkkinen, K., Roslin, T., 2013. Quantifying beetle-mediated effects on gas fluxes from dung pats. PloS One 8(8), e71454. http://dx.doi.org/10.1371/journal.pone.0071454

Philips, K., 2011. The evolutionary history and diversification of dung beetles, in: Simmons, L., Ridsdill-Smith, J., (Eds.), Ecology and Evolution of Dung Bettles. Wiley-Blackwell, Chichester, pp. 21-46.

Ripa, S., Rojas, P., Velasco, G., 1995. Releases of biological control agents of insect pest on Easter Island (Pacific Ocean). Entomophaga 40, 427-440. http://dx.doi. org/10.1007/BF02373730

Robertson, G.P., Swinton, S.M., 2005. Reconciling agricultural productivity and environmental integrity: A grand challenge for agriculture. Frontiers in Ecology and the Environment 3(1), 38-46. http://dx.doi.org/10.1890/15409295(2005)003[0038:RAPAEI]2.0.CO;2

Scholtz, C.H., Davis, A.L.V., Kryger, U., 2009. Evolutionary Biology and Conservation of Dung Beetles. Pensoft, SofiaMoscow, Bulgaria.

Sergio, F., Newton, I., Marchesi, L., Pedrini, P., 2006. Ecologically justified charisma: Preservation of top predators delivers biodiversity conservation. Journal of Applied Ecology 43(6), 1049-1055. http://dx.doi.org/10.1111/ j.1365-2664.2006.01218.x

Simmons, L., Ridsdill-Smith, J., 2011. Ecology and Evolution of Dung Beetles. Wiley-Blackwell. Chichester, United Kingdom.

Slade, E., Mann, D., Villanueva, J., Lewis, O., 2007. Experimental evidence for the effects of dung beetle functional group richness and composition on ecosystem function in a tropical forest. Journal of Animal Ecology 76, 1094-1104. http://dx.doi.org/10.1111/j.13652656.2007.01296.x

Smith, A., 2015. Checklist of the Scarabaeoidea of southern South America. http://museum.unl.edu/research/ entomology/SSSA/SSSA-Guide/SSSA-GenericGuide. html (acceso, 21.09.2015).

Smith, A., Skelley, P., 2007. A review of the Aphodiinae (Coleoptera: Scarabaeidae) of southern South America. Zootaxa 1458, 1-80.

Smith, P., Martino, D., Cai, Z., Gwary, D., Janzen, H., Kumar, P., McCarl, B., Ogle, S., O’Mara, F., Rice, C., Scholes, B., Si- rotenko, O., Howden, M., McAllister, T., Pan, G., Romanenkov, V., Schneider, U., Towprayoon, S., Wattenbach, M., Smith, J., 2008. Greenhouse gas mitigation in agriculture. Philosophical Tansactions of the Royal Society of London. Series B, Biological Sciences 363(1492), 789813. http://dx.doi.org/10.1098/rstb.2007.2184

Steffen, W., 2010. Observed trends in Earth System behavior. Wiley Interdisciplinary Reviews: Climate Change 1(3), 428-449. http://dx.doi.org/10.1002/wcc.36

Steinfeld, H., Gerber, P., Wassenaar, T., Castel, V., Rosales, M., de Hann, C., 2006. Livestock's long shadow: Environmental issues and options. Food and Agriculture Organization of the United Nations (FAO). http://www.fao.org/docrep/010/a0701e/a0701e00.htm (acceso, 21.09.2015).

Sutton, G., Bennett, J., Bateman, M., 2014. Effects of ivermectin residues on dung invertebrate communities in a UK farmland habitat. Insect Conservation and Diversity 7(1), 64-72. http://dx.doi.org/10.1111/icad.12030

Tilman, D., Reich, P.B., Knops, J.M.H., 2006. Biodiversity and ecosystem stability in a decade-long grassland experiment. Nature 441(7093), 629-632. http://dx.doi. org/10.1038/nature04742

Verdú, J.R., Numa, C., Lobo, J.M., Martínez-Azorín, M., Galante, E., 2009. Interactions between rabbits and dung beetles influence the establishment of Erodium praecox. Journal of Arid Environments 73, 713-718. http://dx.doi. org/10.1016/j.jaridenv.2009.02.008

Wall, R., Beynon, S., 2012. Area-wide impact of macrocyclic lactone parasiticides in cattle dung. Medical and Veterinary Entomology 26, 1-8. http://dx.doi.org/10.1111/ j.1365-2915.2011.00984.x

Waterhouse, D., 1974. The biological control of dung. Scientific American 230, 100-109.

Wratten, S.D., Gillespie, M., Decourtye, A., Mader, E., Desneux, N., 2012. Pollinator habitat enhancement: Benefits to other ecosystem services. Agriculture, Ecosystems \& Environment 159, 112-122. http://dx.doi.org/10.1016/j. agee.2012.06.020

Yamada, D., Imura, O., Shi, K., Shibuya, T., 2007. Effect of tunneler dung beetles on cattle dung decomposition, soil nutrients and herbage growth. Grassland Science 53, 121-129. http://dx.doi.org/10.1111/j.1744697X.2007.00082.x

Yokoyama, K., Kai, H., Tsuchiyama, H., 1991. Paracoprid dung beetles and gaseous loss of nitrogen from cow dung. Soil Biology and Biochemistry 23(7), 643-647. http:// dx.doi.org/10.1016/0038-0717(91)90077-W

Yoshihara, Y., Sato, S., 2015. The relationship between dung beetle species richness and ecosystem functioning. Applied Soil Ecology 88, 21-25. http://dx.doi. org/10.1016/j.apsoil.2014.12.001

Zunino, M., 1984. Analisi sistematica e zoogeografica della sottofamiglia Taurocerastinae Germain (Coleoptera, Scarabaeoidea: Geotrupidae). Bollettino del Museo Regionale di Scienze Naturali, Torino 2(2), 445-464. 
